\title{
Beyond the Wade-Mingos Rules in Bare Ten- and Twelve-vertex Germanium Clusters: Transition States for Symmetry Breaking Processes
}

\author{
R. B. King, ${ }^{1}$ I. Silaghi-Dumitrescu, ${ }^{2}$ and M. M. Uţă ${ }^{2}$
}

\section{Supporting Information}

Figures 1S, 2S, 3S, 4S, 5S, 6S

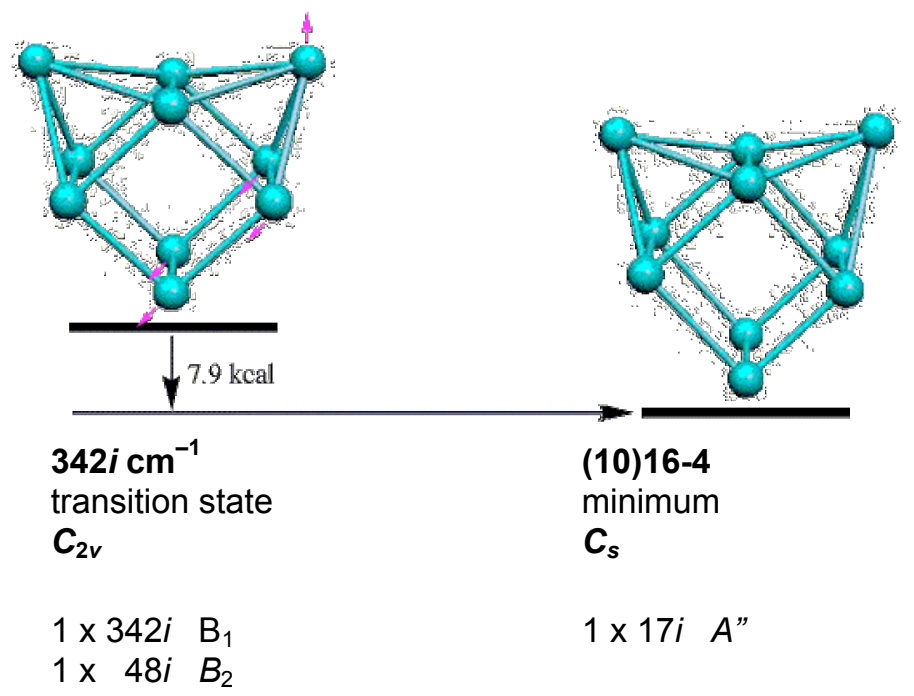

Figure 1S. The distortion of the $C_{2 v}$ bicapped cube transition state in $\mathrm{Ge}_{10}{ }^{4+}$ along the $\mathrm{B}_{1}$ normal mode of the $342 i$ vibrational frequency to give the $C_{s}$ polyhedral structure (10)16-4.

\footnotetext{
${ }^{1}$ University of Georgia

2 Babeş-Bolyai University
} 


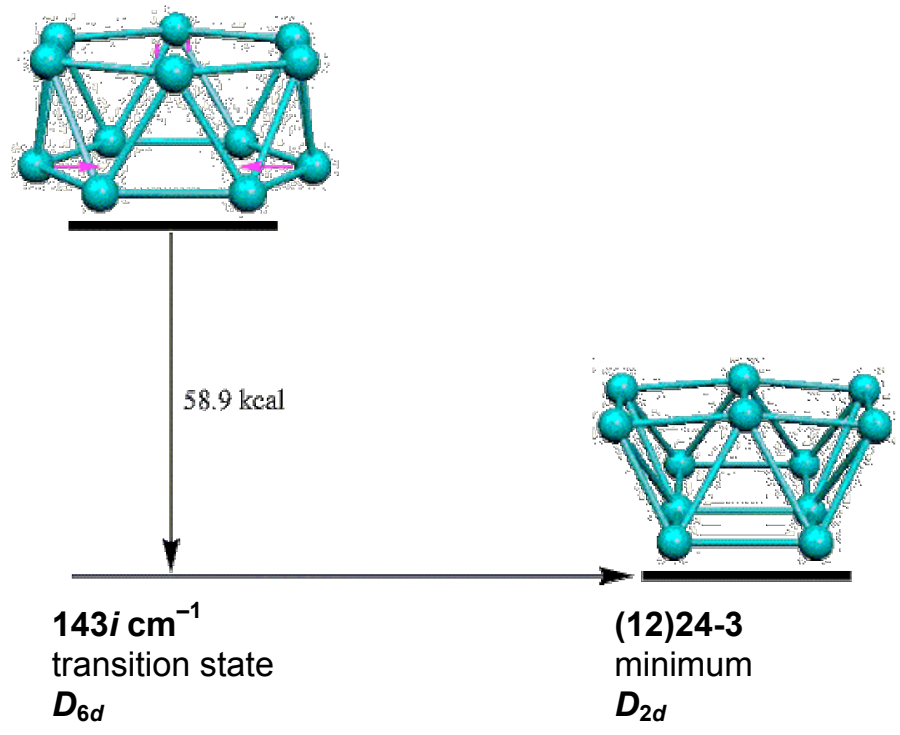

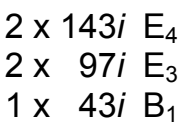

Figure 2S. The distortion of the $D_{6 h}$ hexagonal antiprism transition state in $\mathrm{Ge}_{12}$ along the $\mathrm{E}_{4}$ normal mode of the $143 i$ vibrational frequency to give the $D_{2 d}$ (12)24-3.

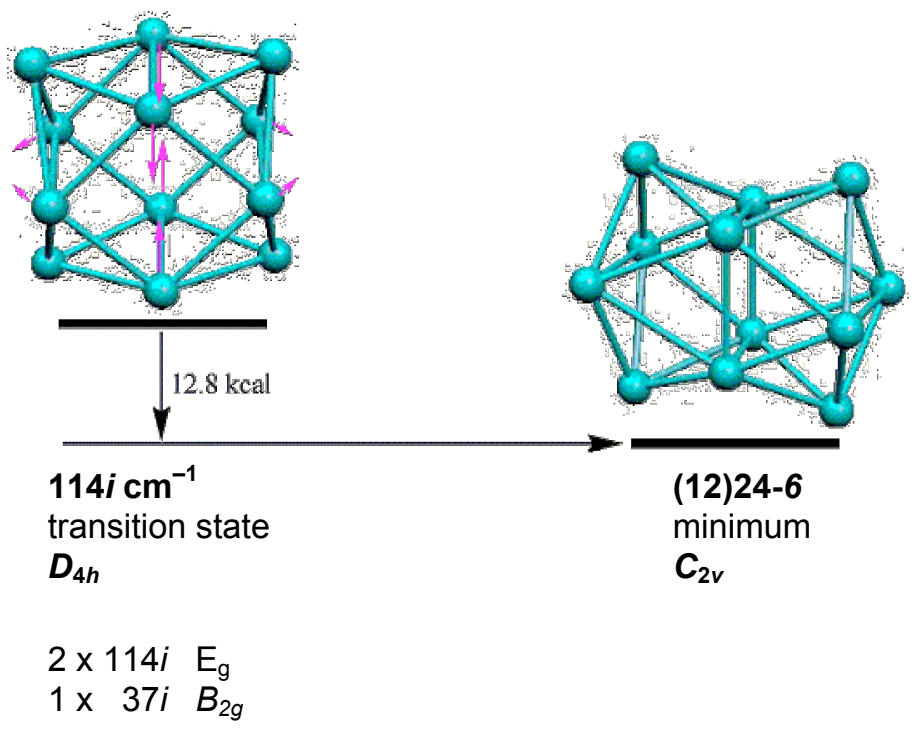

Figure 3S. The distortion of the $D_{4 h}$ tetracapped cube transition state in $\mathrm{Ge}_{12}$ along the $\mathrm{E}_{\mathrm{g}}$ normal mode of the $114 i$ vibrational frequency to give the $C_{2 v}$ (12)24-6. 


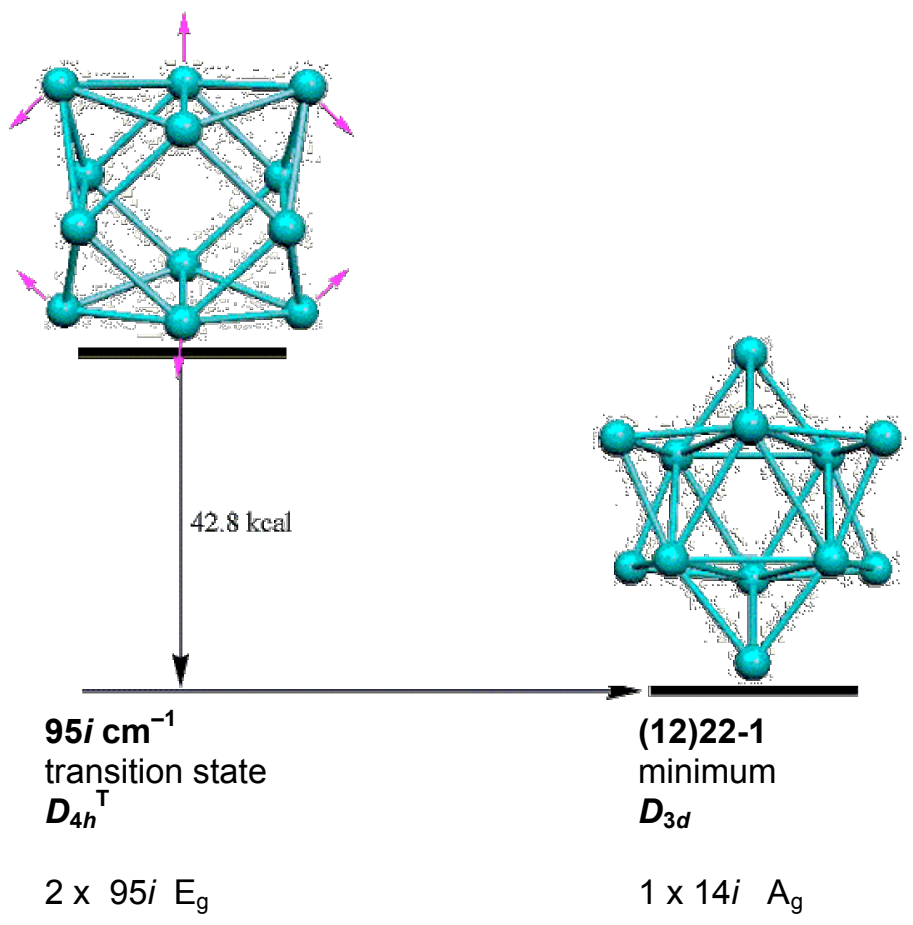

Figure 4S. The distortion of the $D_{4 h}$ tetracapped cube transition state in $\mathrm{Ge}_{12}{ }^{2+}$ along the $\mathrm{E}_{\mathrm{g}}$ normal mode of the $95 i$ vibrational frequency to give the $\mathrm{D}_{3 d}$ hexacapped octahedron (12)22-1. 


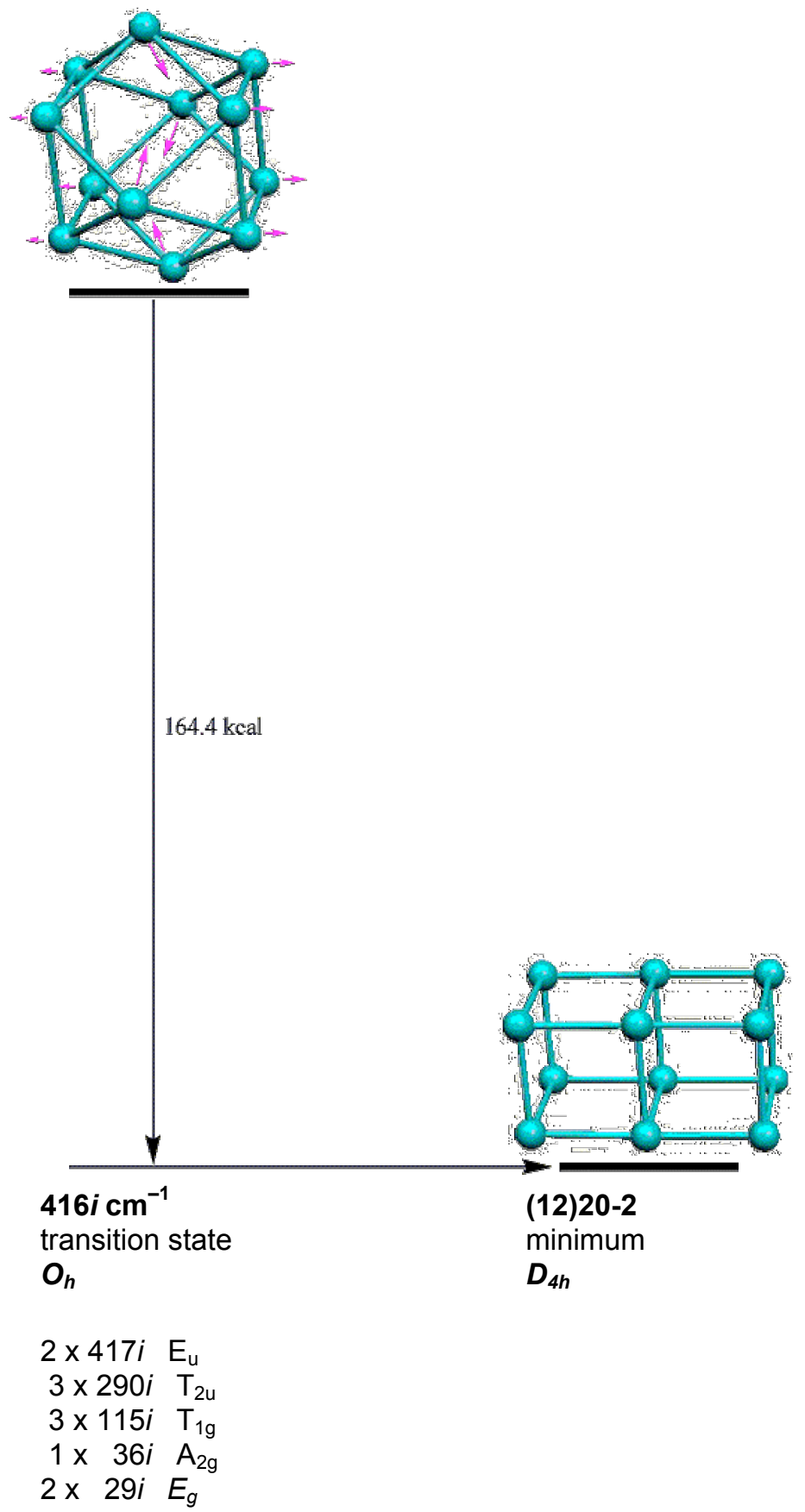

Figure 5S. The distortion of the $O_{h}$ cuboctahedron transition state in $\mathrm{Ge}_{12}{ }^{4+}$ along the $\mathrm{E}_{\mathrm{u}}$ normal mode of the $417 i$ vibrational frequency to give the $D_{4 h}$ double cube (12)20-2. 


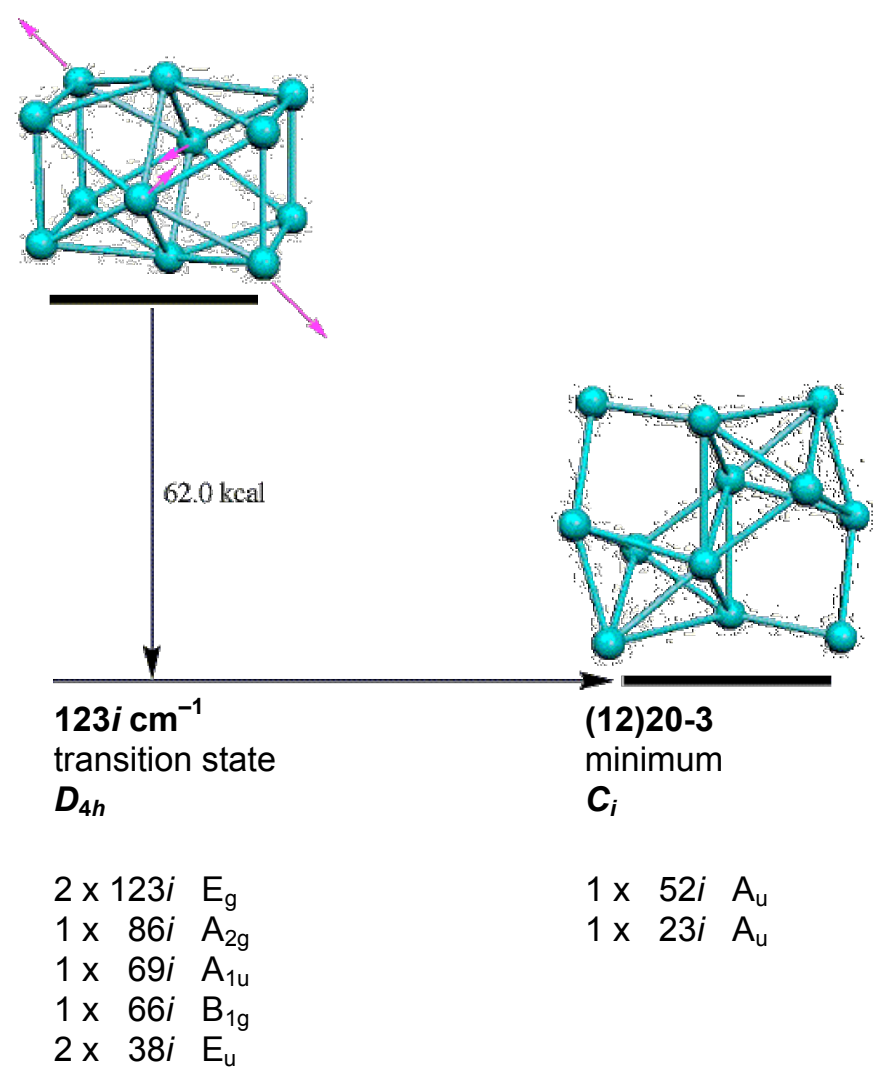

Figure 6S. The distortion of the $D_{4 h}$ double square antiprism transition state in $\mathrm{Ge}_{12}{ }^{4+}$ along the $\mathrm{E}_{\mathrm{g}}$ normal mode of the $123 i$ vibrational frequency to give the $C_{i}$ structure (12)20-3. 